
\title{
3 Research Square \\ Polymorphism in coding region of human CYP2D6 gene contributes to failed radical cure of vivax malaria using primaquine
}

Herong Huang

Dali University School of Basic Medicine

Ying Dong ( $\square$ luxidongying@126.com )

Yanchun Xu

Yunnan Institute of Parasitic Diseases

Yan Deng

Yunnan Institute of Parasitic Diseases

Canglin Zhang

Yunnan Institute of Parasitic Diseases

Shuping Liu

Dali University School of Basic Medicine

Mengni Chen

Yunnan Institute of Parasitic Diseases

Yan Liu

Yunnan Institute of Parasitic Diseases

\section{Research}

Keywords: Plasmodium vivax, Relapse, Cytochrome P450 isoenzyme 2D6, Coding region, Primaquine, Radical Cure, enzyme activity, Phenotypes

Posted Date: May 26th, 2020

DOI: https://doi.org/10.21203/rs.3.rs-19379/v2

License: (c) (1) This work is licensed under a Creative Commons Attribution 4.0 International License.

Read Full License 


\section{Abstract}

Background: So far, it has been confirmed that primaquine is the only medicine could effectively kill the hypnozoites of $P$. vivax. Therefore, World Health Organization (WHO) recommends primaquine as the only anti-relapsing drug for the treatment of vivax malaria patient. However, due to the active component of primaquine for killing $P$. vivax hypnozoite is the 5-hydroxy-primaquine, which has to be catalyzed by the Cytochrome P450, family 2, subfamily D, polypeptide 6 (CYP2D6) enzyme in human hepatocytes, the decrease of CYP2D6 enzyme activity caused by CYP2D6 gene mutation has been considered as an risk factor for the use of primaquine. Based on the analysis of CYP2D6 gene polymorphisms in relapsed vivax malaria patients, this study preliminarily revealed the genetic association between human CYP2D6 genotype and the curative effect of primaquine in vivax malaria.

Methods: Blood samples of vivax malaria cases treated with "chloroquine/primaquine eight-day therapy" from 2014 to 2018 in Yunnan Province were collected. Then, the relapsed cases were determined by identifying the homogeneity of $P$. vivax circumsporozoite protein $₫ p v c s p \llbracket$ gene sequences between the Plasmodiums isolated from patients' first and second infected blood samples. Human genomic DNA was extracted from blood samples, and 9 exons of CYP2D6 gene were amplified by polymerase chain reaction (PCR) and then sequenced. The coding DNA sequence (CDS) of CYP2D6 gene was obtained by splicing after alignment with the wild-type reference sequence. The mutation types of CDS and their association with vivax malaria relapse were analyzed, and the activity phenotype of CYP2D6 isozyme was predicted by the allelic form of CYP2D6 gene.

Results: One hundred and fifty-six blood samples from 75 relapsed cases of vivax malaria and 75 nonrelapsed cases were collected for nested-PCR amplification. Two kinds of nested-PCR products $\triangle$ the CYP2D6 gene fragments containing exon1-4 and exon5-9 (2411bp and 2388bp) 『were obtained from every sample. After splicing and combining these two amplification products, the complete CDS of CYP2D6 (1491 bp) of each sample could be gotten. Clustered CYP2D6 full-CDS of 150 patients, 24 haplotypes (Hap_1 Hap_24) were defined, including 17 haplotypes in relapsed patients and 15 haplotypes in non-relapsed patients. The Hap_6 showed G>C, C>T and G>C base substitutions at c.408, c. 886 and c. 1457, resulting in V136V invariance and the variations of R296C and S486T at $136^{\text {th }}, 296^{\text {th }}$ and $486^{\text {th }}$ amino acid sites. The odds ratio of Hap_6 sequence type was 5.615 (P囚0.05) as compared with the relapse of vivax malaria. For the relapsed cases, the mutation at c.408 of CYP2D 6 gene is $100 \%$ homozygous allele $(C / C, 10 / 10)$, while at $c .886$ is $80 \%$ homozygous allele $(T / T, 8 / 10)$ and $20 \%$ heterozygous allele $(C / T, 2 / 2)$. But the non-relapsed cases only showed mutation heterozygote allele $(\mathrm{C} / \mathrm{T}$, $2 / 2$ ) at c.886 locus. Moreover, most of mutant allele types in relapsed patients were CYP2D 6 *2, and the CYP2D6 enzyme activity of them was predicted as normal metabolizer (NM) by scoring the diploid allele of Hap_6 sequence.

Conclusion: Among the numerous mutations of CYP2D 6 gene, the triple mutations at three loci (c.408, c.886, c.1457) are most closely related to the decreased CYP2D6 enzyme activity, while whether the c.886 locus mutation plays a critical role needs to be further verified by expanded sample size. 


\section{Background}

In recent years, the global malaria epidemic has gradually scaled down, yet the death tolls of malaria remain high, and the number of malaria cases in certain areas has rebounded [1]. At the same time, multiple technological limitations pose daunting challenges to the elimination of malaria, such as the difficulty in identifying low-density Plasmodium infection [2], the population expansion of drug resistant Plasmodium [3-4], and the lack of variety in antimalarial drug [5]. Although Chinese local cases (without a travel history to malaria epidemic areas outside China within the last 30 days before the onset of malaria were defined as local cases) of malaria have not been reported since 2016 [6], China still bears the burden of number large malaria cases imported from abroad every year. In Chinese history, Yunnan Province experienced a large-scale malaria epidemic and was once the hardest-hit area of malaria. As long as Africa and Southeast Asia are the main sources of imported malaria cases in China [7], Yunnan Province which located in southwest China bordering with Myanmar, Laos and other Southeast Asian countries (Fig.1) is still facing a greater risk of malaria import nowadays, especially the vivax malaria. From 2011 to $2018,50.7 \%$ of the imported malaria cases in Yunnan Province are caused by $P$. vivax [8]. Therefore, the effective eradication of malaria in Yunnan Province calls for the strict enforcement of eliminating the source of $P$. vivax and blocking its transmission.

So far, primaquine is the only medication proved to be effective in eradication of the hypnozoites of $P$. vivax, so World Health Organization (WHO) recommended it as the only available antimalarial drug for the clinicians treatment of relapsing forms of malaria [9]. However, the active component in primaquine for killing $P$. vivax hypnozoite, 5-hydroxy-primaquine, requires the catalysis of Cytochrome P450, family 2, subfamily D, polypeptide 6 (CYP2D6) enzyme in human hepatocytes [10-12]. CYP2D6, also known as debrisoquine4-hydroxylase, is an isozyme belonging to the Cytochrome P450 (CYP450) superfamily and an important phase I drug metabolizing enzyme in the human body, containing heme-thiolate ligation as active site. Moreover, CYP2D6 is also the only enzyme in the CYP450 superfamily that cannot be induced by inducers in the environment and it has the features of low capacity and high affinity. Even though CYP2D 6 only accounts for $4 \%$ of the total P450 enzyme protein in the liver, it dominates $\sim 30 \%$ of the metabolism of commonly used drugs, including primaquine [12-14]. Studies have found that the heterogeneity of CYP2D6 activity is predominantly governed by its genetic variation [15], and the decreased CYP2D 6 isoenzyme activity caused by genetic polymorphism has become an adverse factor aggravating the risk of primaquine use. The undermined CYP2D6 enzyme activity would obstruct the generation of 5-hydroxy-primaquine, making large doses or repeated use of primaquine a common remedy for the declined efficacy of primaquine in the treatment. Meanwhile, primaquine can cause life threatening hemolysis in humans with Glucose-6-Phosphate Dehydrogenase (G6PD) deficiency, so patients with G6PD deficiency may be inflicted by dual hazards including low efficacy of anti-relapse treatment using primaquine and the continuously hemolysis induced by repeated use of primaquine [9,16-17]. Owing to the dismal fact that the distribution of patients with defective CYP2D6 is found to be highly coincident with those with G6PD-deficiency, which happened to be in the endemic area of vivax malaria [18-19], the researchers should bear in mind to acquire the patient's G6PD gene type as well as CYP2D 6 enzyme activity when conducting the anti-relapse treatment of vivax malaria $[16,19]$.WHO 
defines that G6PD deficiency, impaired CYP2D6 function, pregnant or lactating women, and young infants are the population not suitable for primaquine treatment $[12,16]$.

The measurement methods of CYP2D6 enzyme activity include phenotype observation and genotype analysis prediction. Compared with phenotype measurement, the prediction method based on CYP2D6 genotype has the following advantages [20-21]. Firstly, genotypes do not change in one's life, and are not affected by environment or physical factors [21-22]. Secondly, genotyping only requires one specimen and can be completed before medication treatment, so it has convenience and foresightedness [23]. In addition, human CYP2D6 genes can be easily sequenced to determine the mutation types of CYP2D6 alleles [24-25]. Therefore, it is more stable and easy-to-handle for batch experiment to predict the phenotype of CYP2D6 enzyme activity by genotyping than another method [20,26-30]. The prediction is based on the locus status of CYP2D6 gene [15], or the allelic combination form of zygote [31-32]. The identified genotypes consist of alleles with complete function, reduced function or loss of function, and the indications of CYP2D6 enzyme activity include Poor metabolizer (PM), Intermediate metabolizer (IM), Normal metabolizer (NM) and Ultra-rapid metabolizer (UM), which cover various active levels of CYP2D6 enzymes from the ultra-fast to the non-metabolic[30,32]. However, more than 80 CYP2D6 alleles have been defined by the Cytochrome P450 Nomenclature Committee at http://www.cypalleles.ki.se [25]. Genotype analysis and prediction method calls for further optimization in precision and systematicness [33].

Gene mutations of the enzymes related to drug metabolism not only affect drug efficacy, but can even reverse the effect of drug leading to toxicity [16]. Therefore, understanding the specific CYP2D6 enzyme activity in different individuals is the prerequisite to ensure radical treatment of vivax malaria with personalized primaquine dose $[30,34]$. However, despite the frequent use of primaquine in malaria endemic areas of Yunnan, no systematic survey about the types of G6PD deficiency and genetic polymorphisms of CYP2D6 has been conducted. Thus, the appropriate population for primaquine treatment is yet to be clarified. In order to improve gene prediction method of CYP2D 6 and provide safety cure of $P$. vivax by using primaquine at the molecular level, this study intends to investigate the genetic relationship between human CYP2D6 genotype and the declined efficacy of primaquine in the radical cure of $P$. vivax.

\section{Materials And Methods}

\section{Subjects and blood sample}

Blood samples of diagnosed and reported vivax malaria cases were continuously collected in Yunnan Province from 2014 to 2018 (Samples are used for re-check of diagnosis provided by the Centers for Disease Control and Prevention of each prefecture and city in Yunnan Province). The mono-infection of $P$. vivax requires the double parasitically confirmation by both microscopy examination and Plasmodium $18 \mathrm{~S}$ rRNA gene detection (PCR) by Yunnan Province Referencet Laboratory(YNRL). Dried blood spots samples for genetic analysis were derived from $0.6 \mathrm{ml}$ of venous blood, then put it in a drying tube and 
stored at $-80^{\circ} \mathrm{C}$ before DNA extraction. The cases were divided into two groups: relapsed and nonrelapsed. All vivax malaria patients spent three days in the county hospital and was given three days of oral Chloroquine therapy (total $1550 \mathrm{mg}$ ), after which was given an 8-day course of Primaquine (22.5 $\mathrm{mg} /$ day). The relapsed cases consisted of patients who relapsed after above-mentioned treatment, and their infection should be caused by the same Plasmodium in two samples detected by sequencing of $P$. vivax circumsporozoite protein ( $p v c s p$ ). A total of 75 relapsed cases were confirmed in Yunnan Province from 2014 to 2018. Non-relapsed cases were randomly selected from vivax malaria cases of 2018 . To avoid bias caused by different sample size, the two groups had equal number of cases, and the finally determined total number of experimental samples is 150 . The infection sources of vivax malaria cases were confirmed according to epidemiological investigation, i.e, those without a travel history to epidemic areas outside Yunnan Province within the last 30 days before the onset of malaria were defined as local cases; those who have a history of travelling to epidemic regions, such as Myanmar and Africa, were regarded as imported cases.

\section{Extraction of human genomic DNA}

Three dried blood spots, each with a diameter of $5 \mathrm{~mm}$, was taken, and human genomic DNA was extracted using QIAgen Mini Kit kit (Germany, QIAamp Company's DNA Mini Kit), according to the manufacture's instruction. The extracted DNA was stored at $-20^{\circ} \mathrm{C}$ for later use.

\section{PCR amplification of CYP2D6 gene fragment}

The polymerase chain reaction (PCR) amplification primers of CYP2D6 gene were designed by using GenBank (https://www.ncbi.nlm.nih.gov/gene/), reference sequence (ID囚NC_000022.11) was used as template, following the reaction conditions in previous references $[27,35]$. The primers for first-round nested PCR covering the coding region of CYP2D6 gene exons1 4were:5'-CCAGTGACAGATAAGGGTGC-3' and 5'-GACGTGGATAGGAGGTACAGAG-3'; the primers for second-round nested PCR were: 5'GGTGACTTCTCCGACCAGG-3' and 5'-TTCCCAAACCCATCTATGC-3'. The final amplification region was 42131088 42128678, and the expected fragment length of the product was $2411 \mathrm{bp}$. The primers that cover exons5 9 of CYP2D6 gene were: 5'-GCCGACTGAGCCCTGGGAGGTAGGTA-3' and 5'GCTGGGGCCTGAGACTT-3. The amplification area was $42126035 \sim 42128422$, and the amplification products had an expected fragment length of $2388 \mathrm{bp}$. For all the PCR reaction systems, we used $2.6 \mu \mathrm{l}$ DNA template, $14.0 \mu \mathrm{l} 2 \times$ Taq PCR hybrid system (QIAGEN, Germany), $0.7 \mu \mathrm{l}$ upstream primer (20umol / $\mathrm{L}), 0.7 \mu \mathrm{l}$ downstream primers (20umol / L). The total volume was made up to $25.0 \mu \mathrm{l}$ with ddH2O. PCR reaction conditions were clarified as follows: 92 to $94^{\circ} \mathrm{C}$ for $2-5$ mins; 92 to $94^{\circ} \mathrm{C}$ for $10-30$ s, 50 to $56^{\circ} \mathrm{C}$ for $15-30 \mathrm{~s}, 68$ to $72^{\circ} \mathrm{C}$ for 2 to $3 \mathrm{~min}, 35$ cycles; 68 to $72^{\circ} \mathrm{C}$ for 7 mins. The triplicated parallel repetition was adopted for each PCR reaction. The amplified products were observed using $1.5 \%$ agarose gel electrophoresis. Then the positive amplification products were sent to Shanghai Meiji Biomedical Technology Co, Ltd. for sequencing using the dideoxy chain-termination method. Only the sequences showed identical at least in two tests were used for subsequent analysis.

\section{Mutation analysis of CYP2D6 coding region}


The sequencing results were aligned using DNAStar v5.10 and BioEdit v7.0.9.0 software. The obtained all DNA sequences were assessed by the Basic Local Alignment Search Tool (BLAST, http://blast.ncbi.nlm.nih.gov/Blast.cgi) in the NCBI platform to verify whether them belong to CYP2D6 gene. When DNA sequences were BLAST with NC_000022.11, these sequences with Identifications equals $100 \%$ and the Query cover were above $99 \%$ were considered as CYP2D6 gene sequence of human. The DNA sequence of exon1-9 region of CYP2D6 gene was determined and spliced into the CYP2D6 CDS in the order of exon 1 to exon9.MEGA v5.04 software was used to align the CDS of each case with the wildtype sequence to confirm the loci of missense mutation and synonymous mutation. DnaSP v6.11.01 software was used to identify missense mutation and synonymous mutation locus in each CDS, and the parameters of DNA diversity index $(\pi)$ and expected heterozygosity $(\mathrm{He})$ were calculated [36]. The allele form of CYP2D6 gene coding region was determined by reference [27-29].

SPSS software (version 21.0; IBN; Chicago; IL) was used to conduct chi-square test on the frequency of haplotype co-existed in relapsed and non-relapsed patients. The odds ratio (OR) value of haplotypes with statistical significance were calculated by compared to the relapse rate of $P$. vivax, and the significance level was 0.05 . OR $\otimes 1$ indicates the haplotype is a protective factor and $\mathrm{OR}>1$ means a risk factor.

\section{Prediction of CYP2D6 enzyme activity}

The mutation loci (base substitution loci) of CYP2D6 gene were used to directly predict the enzymatic activity as one of four phenotypes, PM, IM, NM or UM, in accordance with previously introduced methods [20-21]. The allelic form of the coding region of CYP2D6 gene was identified according to the methods described by Gaedigk et al [22], Sistonen et al [27] and Zanger et al [29]. Among them, CYP2D6* 1 is wildtype allele; * 2 has base substitutions at c. 408, c. 886 and c. 1457 . Nevertheless, both two are the same functional normal allele type. * 10 and $* 41$ are the allele of decreased enzyme activity caused by the $C>$ $T$ and the $\mathrm{G}>\mathrm{A}$ base substitution at c. 100 and c. $1023+36$, respectively.

Subsequently, we referred to previously described methods $[30,33]$ to assign the activity score of the allelic form of CYP2D 6 gene. Among them, the mutations at c. 408, c. 886 and c. 1457 have no evidence to be related to the decrease of enzyme activity, so the three loci cannot be included in the activity score system. The loci that can be included in the activity score system were c. 100 and c. $1023+36$, and their wild type alleles are assigned 1.0. The mutant alleles of c.100 and intron c. $1023+36$ scored 0.25 and 0.5 , respectively. For the event of multiple loci in the genotype, the enzyme activity score is calculated as the sum of the scores of the zygotes included. When the diploid score $x$ falls within the interval of $0.0,0<x<$ $1.25,1.25 \leq x \leq 2.25,>2.25$, it indicates that CYP2D6 isozyme activity has the phenotype of PM, IM, NM and $U M$, respectively.

\section{Results}

\section{Sample information and PCR amplification of human CYP2D6 gene}


A total of 75 relapsed cases of $P$. vivax were enrolled. Among them, 71 cases, 2 cases and 2 cases had one, two and three relapse events, respectively. The basic information of 75 non-relapsed cases is listed in Table 1. The male-female ratio was 2.66:1, and the proportions of these cases imported from Myanmar infection, imported from Africa countries infection, Laos infection and Yunnan indigenous infection were $96.7 \%$ (145/150), 1.3\% (2/150), $0.7 \%(1 / 150)$ and 1.3\% (2/150), respectively. The odds ratio of relapsed was calculated in different age groups and genders. The results showed that only the age groups of 5-20 years old and 21-60 years old had statistical significance ( $P \otimes 0.05)$, which were 0.380 (95\% Cl:

$0.986 \sim 0.146)$ and 2.471 (95\% Cl: 5.417 1.127) respectively, that is, the risk of relapsed of vivax malaria in patients aged 5-20 years old was reduced. The risk of relapsed between the patients ages of 21-60 was 2.471 times higher than that of other age groups. There was no significant correlation between other age groups or gender and the relapsed of vivax malaria.

Table 1 Information of 150 vivax malaria cases whose blood samples successful amplified CYP2D6 gene exon1-9 fragments

\begin{tabular}{lcccccc}
\hline \multirow{2}{*}{ Characteristic } & \multicolumn{5}{c}{ Years } & \multirow{2}{*}{ Total } \\
\cline { 2 - 6 } & 2014 & 2015 & 2016 & 2017 & 2018 & \\
\hline Number & 18 & 27 & 20 & 10 & 75 & 150 \\
1.Relapse times & & & & & & \\
$\quad 0$ & 0 & 0 & 0 & 0 & 75 & 75 \\
1 & 17 & 25 & 19 & 10 & 0 & 71 \\
2 & 0 & 1 & 1 & 0 & 0 & 2 \\
3 & 1 & 1 & 0 & 0 & 0 & 2 \\
2. Age & & & & & & \\
$0-4$ & 0 & 0 & 1 & 2 & 1 & 4 \\
5-20 & 0 & 2 & 4 & 1 & 16 & 23 \\
$21-60$ & 17 & 25 & 15 & 6 & 51 & 114 \\
above 60 & 1 & 0 & 0 & 1 & 7 & 9 \\
3. Gender & & & & & & \\
Male & 16 & 22 & 14 & 6 & 51 & 109 \\
Female & 2 & 5 & 6 & 4 & 24 & 41 \\
4. Infection source & & & & & & \\
Myanmar & 16 & 26 & 18 & 10 & 75 & 145 \\
Africa & 1 & 0 & 1 & 0 & 0 & 2 \\
Laos & 0 & 1 & 0 & 0 & 0 & 1 \\
Yunnan indigenous & 1 & 0 & 1 & 0 & 0 & 2 \\
\hline
\end{tabular}

${ }^{a}$ Years old, ${ }^{b}$ Numbers, ${ }^{c}$ Identified by epidemiological investigation

One hundred and fifty-six blood samples of relapsed cases were collected, and PCR amplification of two segments of human CYP2D6 gene including exons 1-4 and exons5-9 was performed, along with 75 blood samples of non-relapsed cases.

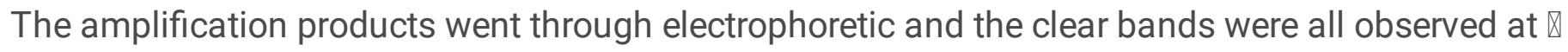
$2000 \mathrm{bp}$, which were considered to be the target bands (Fig.2).

\section{Polymorphism analysis of CYP2D6 gene coding region}


The PCR amplification products of CYP2D6 gene from the relapsed cases and non-relapsed cases were trimmed, and the CDS chains containing complete exon1-9 (total length $=1491 \mathrm{bp}$ ) were obtained from all samples. A total of 150 CDS chains $\llbracket$ Genbank accession number: MT339044-MT339193\ were selected from each of the 75 relapsed case and 75 non-relapsed cases, and were compared with wild-type sequence (NC:000022.11). Base substitutions at 12 loci such as c.31 and c.100 were found (Table 2). The proportion of the third and the first base substitution of the codon triplet was $41.7 \%(5 / 12)$, and the proportion of the second base substitution was $16.6 \%(2 / 12)$. There were 7 missense mutation loci and 5 synonymous mutation loci. The mutation loci of relapsed cases accounted for $91.7 \%(11 / 12)$, and the mutation loci of non-relapsed cases accounted for $66.7 \%(8 / 12)$ (Table 2)

Table 2 Polymorphism Comparison of Relapse cases and Non-relapse cases in the coding domain of CYP2D6 Genes from $1^{\text {st }}$ aa to $497^{\text {th }}$ aa

$\begin{array}{ll}\text { Relapse cases } & \text { Non-relapse cases }\end{array}$

\begin{tabular}{|c|c|c|c|c|c|c|}
\hline Loci & Codon change $^{\mathrm{a}}$ & Amino acid change & & Loci & Codon change $^{a}$ & Amino acid change \\
\hline c.31 & GTG $\square A T G$ & V11M & & -- & -- & -- \\
\hline c. 100 & ССА $\square$ TCA & P34S & - & c. 100 & ССА $\square \mathrm{TCA}$ & P34S \\
\hline C. 271 & CTG $\square$ ATG & L91M & & c. 271 & CTG $\square T \mathrm{TG}$ & L91L \\
\hline c.281 & $\mathrm{CAC} \square \mathrm{C} G \mathrm{C}$ & H94R & & -- & -- & -- \\
\hline C. 294 & $\mathrm{ACC} \square \mathrm{ACG}$ & T98T & & c. 294 & $\mathrm{ACC} \square \mathrm{ACG}$ & T98T \\
\hline c. 297 & $\mathrm{GCC} \square \mathrm{GCT}$ & A99A & & -- & -- & -- \\
\hline c.336 & ТТС $₫ T T$ & $\mathrm{~F} 112 \mathrm{~F}$ & & c.336 & ТТС $\square T T$ & $\mathrm{~F} 112 \mathrm{~F}$ \\
\hline C. 408 & GTC $₫ \mathrm{GTG}$ & V136V & & c. 408 & GTC $\square \mathrm{GTG}$ & V136V \\
\hline c. 505 & $\mathrm{GGT} \square \boldsymbol{A G T}$ & G169S & & -- & -- & -- \\
\hline-- & -- & -- & & c. 801 & $\mathrm{CCC} \square \mathrm{CC} A$ & P267P \\
\hline c.886 & $\mathrm{CGC} \square \mathrm{TGC}$ & R296C & & c.886 & $\mathrm{CGC} \square \mathrm{TGC}$ & R296C \\
\hline c. 1457 & $\mathrm{AGC} \square \mathrm{ACC}$ & S486T & & c.1457 & $\mathrm{AGC} \square \mathrm{A} C \mathrm{C}$ & S486T \\
\hline
\end{tabular}

\section{a DNA base highlighted in bold are where the SNP occur}

\section{Polymorphism of haplotypes}

A total of 24 CYP2D6 haplotypes (Hap_18Hap_24) were clustered amongst the 150 vivax malaria samples examined: 17 haplotypes were observed in the sequences of relapsed patients, $\pi$ and He equal to 0.0015 and 0.8191 , respectively. 15 haplotypes were observed in the sequences of non-relapsed patients $(\pi=0.0014$ and $\mathrm{He}=0.8065)$. There are 8 haplotypes both in the sequences of relapsed and non-relapsed patients, including Hap_2, Hap_3, Hap_4, Hap_5, Hap_6, Hap_7, Hap_14 and Hap_17. Haplotype and frequency were shown in Fig. 3. Among them, Hap_3 was wild-type sequence, and all the rest were mutant sequence. Hap_2 accounted for the largest proportion of $36.6 \%$ (55/150), followed by 15.3\% for Hap_3 (23/150), 8.6\% for Hap_4 (13/150), 6.6\% for Hap_5 (10/150), 8.0\% for Hap_6 (12/150), 
6.6\% for Hap_7 (10/150) and 4.6\% for Hap_21 (7/150). The proportions of Hap_1, Hap_8, Hap_9, Hap_10, Hap_12, Hap_13, Hap_15, Hap_16, Hap_18, Hap_19, Hap_20, Hap_22, Hap_23, and Hap_24 were as low as $0.7 \%(1 / 150)$. The proportions of Hap_11, Hap_14 and Hap_17 haplotypes were 1.3\% (2/150).

\section{Haplotypes of CYP2D6 gene coding region and their association with relapse}

Six haplotype sequences with sequence number $>1$ that simultaneously existed in patients with and without recurrence was selected to calculate the odds ratio (Table 3). Among them, only two haplotypes,

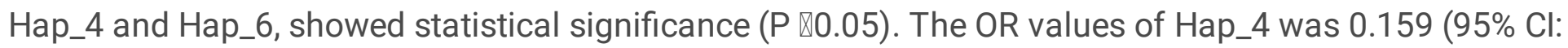
0.746 0.034) and Hap_6 was 5.615 (95\% Cl: 26.577 1.186). So, it demonstrated that the occur of Hap_4 indicates reduced the risk of relapsing vivax malaria. By contrast, the risk of relapsing vivax malaria in Hap_6 was about 5.615-fold higher than in other haplotypes. The OR values of Hap_2, Hap_3, Hap_5 and Hap_7 showed no statistical significance $(P>0.05)$ (Table 3).

Table 3 Analysis of the relationship between haplotypes and relapse

\begin{tabular}{lcccccc}
\hline Hap & Relapse cases(n) & Non-relapse cases(n) & OR & \multicolumn{2}{c}{$95 \% C I$} & \multirow{2}{*}{ P } \\
\cline { 5 - 6 } & & & & Upper limit & Low limit & \\
\hline Hap_2 & 28 & 27 & 1.059 & 2.058 & 0.545 & $0.865^{\mathrm{a}}(\mathrm{NS})$ \\
Hap_3 & 8 & 15 & 0.478 & 1.206 & 1.189 & $0.113^{\mathrm{a}}(\mathrm{NS})$ \\
Hap_4 & 2 & 11 & 0.159 & 0.746 & 0.034 & $0.009^{\mathrm{a}}(\mathrm{S})$ \\
Hap_5 & 8 & 2 & 4.358 & 21.258 & 0.894 & $0.050^{\mathrm{a}}(\mathrm{NS})$ \\
Hap_6 & 10 & 2 & 5.615 & 26.577 & 1.186 & $0.016^{\mathrm{a}}(\mathrm{S})$ \\
Hap_7 & 7 & 3 & 2.471 & 9.944 & 0.614 & $0.190^{\mathrm{a}}(\mathrm{NS})$
\end{tabular}

n: number of cases; NS: not significant; S: significant; a: Chi-square test

\section{Enzyme activity prediction of significant haplotypes and their diploids}

The enzyme activity of Hap_6 and Hap_4 genotypes was predicted for they were proved to play an important role in the recurrence. There were 12 CDS chains in Hap_6 (Table 4) from 10 relapsed patients and 2 non-relapsed patients. The diploids in relapsed cases presented 4 genotypes, including $* 1 / * 2$, $\star 2 / * 2, * 2 / * 41$ and $* 1 / * 41$. Among the mutation loci of c. 408, c. $886, c .1023+36$ and c. 1457 , only the mutations at c.886 and c. $1023+36$ were diploid alleles. Regardless of the diploid of $c .886$ being mutant heterozygosity $(\mathrm{C} / \mathrm{T})$ or mutant homozygosity $(\mathrm{T} / \mathrm{T})$, as long as it co-exists with the mutant heterozygosity of c.1023+36, the genotype score of CYP2D6 couldn't reach the full score of 2.0. In this case, the score was 1.5 and the enzyme activity of CYP2D6 was "NM" (Table 4). The genotypes of * $1 /{ }^{*} 2$ and $* 1 / * 41$ were found in the non-relapsed cases. However, $* 1 / * 41$ was also determined in the relapsed cases and reached the genotype score of 1.5. It was predicted to be at "NM" level in terms of enzyme activity, along with the $* 1 / * 2$ genotype showing mutant heterozygous $(\mathrm{C} / \mathrm{T})$ only at c.886 and the full genotype score (Table 4). 
Table 4 Prediction of enzyme activities of Hap-6 and Hap-4 genotypes

A. Hap_6

\begin{tabular}{|c|c|c|c|c|c|c|c|c|}
\hline \multirow[t]{2}{*}{ Genotypes } & \multirow[t]{2}{*}{ Relapse times } & \multirow[t]{2}{*}{ No. of samples } & \multicolumn{4}{|c|}{ Mutation loci and their diploid } & \multicolumn{2}{|c|}{ Caudle's method } \\
\hline & & & c.408 & c.886 & c. $1023+36^{\mathrm{a}}$ & c.1457 & Score of diploid & Degree \\
\hline NC_000022.11 & -- & -- & $\mathrm{G} / \mathrm{G}$ & $\mathrm{C} / \mathrm{C}$ & $\mathrm{G} / \mathrm{G}$ & $\mathrm{G} / \mathrm{G}$ & 2 & $\mathrm{NM}$ \\
\hline$* 1 / * 2$ & 0 & 1 & $\mathrm{C} / \mathrm{C}$ & $\mathrm{C} / \mathrm{T}$ & $\mathrm{G} / \mathrm{G}$ & $\mathrm{C} / \mathrm{C}$ & 2 & NM \\
\hline$* 1 / * 41$ & 0 & 1 & $\mathrm{C} / \mathrm{C}$ & $\mathrm{C} / \mathrm{T}$ & $\mathrm{G} / A$ & $\mathrm{C} / \mathrm{C}$ & 1.5 & NM \\
\hline$* 1 / * 2$ & 1 & 1 & $\mathrm{C} / \mathrm{C}$ & $\mathrm{C} / \mathrm{T}$ & $\mathrm{G} / \mathrm{G}$ & $\mathrm{C} / \mathrm{C}$ & 2 & NM \\
\hline$* 2 / * 2$ & 1 & 5 & $\mathrm{C} / \mathrm{C}$ & $T / T$ & G/G & $\mathrm{C} / \mathrm{C}$ & 2 & NM \\
\hline$* 2 / * 41$ & 1 & 3 & $\mathrm{C} / \mathrm{C}$ & $T / T$ & $\mathrm{G} / \boldsymbol{A}$ & $\mathrm{C} / \mathrm{C}$ & 1.5 & NM \\
\hline$* 1 / * 41$ & 1 & 1 & $\mathrm{C} / \mathrm{C}$ & $\mathrm{C} / T$ & $\mathrm{G} / \boldsymbol{A}$ & $\mathrm{C} / \mathrm{C}$ & 1.5 & NM \\
\hline
\end{tabular}

B. Hap_4

\begin{tabular}{|c|c|c|c|c|c|c|c|c|}
\hline \multirow[t]{2}{*}{ Genotypes } & \multirow[t]{2}{*}{ Relapse times } & \multirow[t]{2}{*}{ No. of samples } & \multicolumn{4}{|c|}{ Mutation loci and their diploid } & \multicolumn{2}{|c|}{ Caudle's method } \\
\hline & & & c.100 & c.408 & -- & c.1457 & Score of diploid & Degree \\
\hline NC_000022.11 & -- & -- & $\mathrm{C} / \mathrm{C}$ & $\mathrm{G} / \mathrm{G}$ & -- & $\mathrm{G} / \mathrm{G}$ & 2 & NM \\
\hline$* 10 / * 10$ & 0 & 2 & $T / T$ & $C / C$ & -- & $C / C$ & 0.5 & IM \\
\hline$* 1 / * 10-1$ & 0 & 3 & $\mathrm{C} / \mathrm{T}$ & $C / C$ & -- & $\mathrm{G} / C$ & 1.25 & NM \\
\hline$* 1 / * 10-2$ & 0 & 6 & $\mathrm{C} / T$ & $C / C$ & -- & $C / C$ & 1.25 & NM \\
\hline$* 1 / * 10-2$ & 1 & 2 & $\mathrm{C} / \mathrm{T}$ & $C / C$ & -- & $C / C$ & 1.25 & NM \\
\hline
\end{tabular}

a:Intronic position c.1023+36aIntron 2988 ahas been considered as defining allele $* 41$ because the predictivity of this mutation for impaired enzyme function has been demonstrated recently

The 13 CDS chains belonging to Hap_4 was found in 2 relapsed and 11 non-relapsed patients (Table 4). In the relapsed cases, only one diploid genotype was found, the *1/*10-2. The mutations of $* 1 / * 10-2$ at loci c. 100, c. 408 and c.1457 were heterozygosity (C/T), homozygosity $(C / C)$ and homozygosity $(C / C)$, respectively. Such pattern resembled the genotype of $54.6 \%(6 / 11)$ of non-relapsed cases, and reached the genotype score of 1.25. The CYP2D6 enzyme activity based on this score was predicted at "NM" level (Table 4). The remaining 5 non-relapsed cases were classified as the genotypes of $* 10 / * 10$ and $* 1 / * 10-1$. The diploids of genotypes $* 10 / * 10$ at the mutation loci of c. 100 , c.408 and c. 1457 were all mutant homozygous $(T / T),(C / C)$ and $(C / C)$, with the lowest genotype score being 0.5 . It was predicted that the activity of CYP2D 6 enzyme is "IM" (Table 4). In 3 cases of *1/*10-1 genotype, mutation heterozygosity $(\mathrm{C} / \mathrm{T})$ was found at the $\mathrm{c} .100$, and the genotype score was 1.25 , suggesting that its CYP2D6 enzyme activity was predicted as "NM" level (Table 4).

\section{Discussion}

The 4383-bp-length CYP2D 6 gene locates on chromosome 22q13.1 and consists of 8 9 exons and 7 8

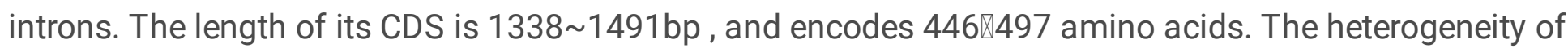
CYP2D6 monooxygenase activity varies from complete dysfunction to ultra-rapid enzyme metabolism according to genotypes of CYP2D6 [16]. Among the 150 identified alleles, multiple mutations, such as CYP2D6*3, CYP2D6*4, CYP2D6*5 and CYP2D6*6, are considered to be associated with compromised CYP2D6 enzyme activity [35,37].

To our knowledge, the current study is the first one that attempts to analyze mutation polymorphism in the whole gene coding region of human CYP2D6 gene, thereby verifying its possible associated with 
impaired CYP2D6 enzyme activity in patients with relapsed vivax malaria events after primaquine treatment in Yunnan Province from 2014 to 2018. Among the 24 haplotypes defined, Hap_2 makes up the largest proportion of the sequences, reaching as high as $36.6 \%(55 / 150)$. This haplotype has multiple mutations at four loci, including c. $100 \mathrm{C}>\mathrm{T}, \mathrm{c} .336 \mathrm{C}>\mathrm{T}, \mathrm{c} .408 \mathrm{G}>\mathrm{C}, \mathrm{c} .1457 \mathrm{G}>\mathrm{C}$. These mutations are different with previous studies which reported that CYP2D6*10 allele is easy to be detected in Asian populations $[35,38]$. CYP2D6*10 allele has multiple mutations at c. $100(\mathrm{C}>\mathrm{T}), \mathrm{c} .408(\mathrm{G}>\mathrm{C})$ and C. $1457(G>C)$, which lacks c. 336C > T compared with Hap_2. This gap might be resulted from the fact that the sequencing of the whole gene coding region is an easier method to detect all base substitutions. However, whether the differences in common alleles of CYP2D6 are worthy of in-depth research is subject to different populations. The population studied in this paper is not random population, but the vivax malaria patients who reside in disease endemic areas, and $50 \%$ of them $(75 / 150)$ relapsed the malaria at once. Thusly, the genotypes of such population may show the selected outcome of human defective genes after the screening of malaria epidemic in an accurate manner [39].

Although it is speculated that the enzyme activity decrease in the heterogeneity of CYP2D6 may be related to the failed therapy of primaquine to kill hypnozoite of $P$. vivax $[9,19,40-42]$, the correlation between different genotypes and the relapse of $P$. vivax is poorly understood. In this study, we found that the genotypes with combined mutations at c. 408, c. 886 and c. 1457 were most closely related to the relapse of vivax malaria $(\mathrm{OR}=5.615, \mathrm{P}<0.05)($ Table 3$)$, and the key to determining vivax malaria relapse may lie in mutation at c. 886 locus. Such findings are not consistent with that of Silvino et al [42], who believes that c. 100, c. 321 and other locus mutations are more closely related to vivax malaria relapse. Such disparity might be attributed to the difference between the two methods used for detecting gene mutation loci. Silvino et al. employed the method of restriction enzyme digestion for individual locus, such as c.100 and c.321, while the whole gene coding region sequencing method used in this study was easier to find locus polymorphism in the gene. Of note, in determining the close association between the multiple mutations at c. 408, c. 886 and c. 1457 and the relapse of vivax malaria, we found the intron c. 1023+36 mutation may play a role in aggravating the decrease of CYP2D6 enzyme activity. In 4 relapsed cases of c. 886 mutation heterozygosity $(\mathrm{C} / \mathrm{T})$ or homozygous mutation $(\mathrm{T} / \mathrm{T})$, mutations were found at c. 1023+36, which was only the mutant heterozygotes from genetic background. This finding concurs with c. 1023+36 mutations found by Silvino et al [42] and Raimundo et al [43] in patients who suffered from failed treatment of vivax malaria. On the other hand, the multiple mutation genotypes at c. 100, c.408 and c. 1457 loci found in this study depicted a unique phenomenon of reducing the relapse risk of vivax malaria $(\mathrm{OR}=0.159, \mathrm{P}<0.05)$, so warrants further analysis and confirmation.

At present, the main methods for predicting CYP2D6 enzyme activity based on gene information include theCYP2D6-locus-mutation-polymorphism-based direct prediction method summarized by Zanger et al [29] and indirect prediction method of genotype score recommended by Gaedigk et al [30] and Caudle et al [33].Because the latter presents the advantage of quantifying the degree of gene mutation and having more abundant genotypic criteria, we used this method to predict and analyze the activity of CYP2D6 enzymes in this study. However, whether the Hap_6 indicating risk status or the Hap_4 serving as protective factors, all the genotypes of them were predicted to be above IM grade. The allele type of most 
relapsed patients was CYP2D6*2, and most of the non-relapsed patients had CYP2D6*10 allele. This result is not in line with that of Brasil et al [41], which reported that most of the alleles in relapsed patients are CYP2D6*10, CYP2D6*39 and so forth. Moreover, it contradicts the clinical observation of relapsed patients in this study. Such conflict might be justified by the reason that prediction method of CYP2D6 enzyme activity used in this study is focus on the whole gene coding region information, thereby takes more alleles into the account and leads to enhanced genotype score. In contrast, Brasil et al detected only a few mutation loci and therefore could miss out the reverse effects of other locus mutations. CYP2D6*2 allele shows heterogeneous phenotypes with normal and abnormal enzyme activity [44], suggesting that identifying the heterogeneous CYP2D6 enzyme activity with similar genotypes is the key to predicate the enzyme activity of CYP2D6 [22, 30], no matter which kind of strategy is used. Meanwhile, the prediction method needs to be further optimized, for it has not ruled out the effect of primaquine resistance in patients with relapsed vivax malaria. Therefore, it is an important aspect deserving of improvement in the prediction method that to eliminate the influence from interfering factors.

In this study, although first-generation sequencing method was used to analyze the polymorphism in the whole coding region of CYP2D6 gene, the strategy of triplicated parallel repetition was adopted for each PCR reaction and the sequencing of its products. Such effort helped eliminate the error rate in single PCR direct sequencing to a certain extent. Meanwhile, the cost-effective sequencing analysis method is more suitable for molecular epidemiological cross-sectional investigation of CYP2D6 gene mutation polymorphism in the endemic areas where primaquine is used to treat vivax malaria. However, there are still some shortcomings in this study. Firstly, the sample size is not large enough, and the low-frequency alleles of CYP2D6 genes detected in the study should be further verified. Secondly, the non-relapsed patients selected are all from 2018 in this study, which is not equivalent to the relapsed patients from four years. The sample bias has a certain influence on the experimental results due to the changes of various factors in different years. In addition, in the study of genetic association between CYP2D6 genotypes and relapsed $P$. vivax, although the identification of relapsed cases had achieved by the comparison of the genetic origin of infected strains, it did not rule out the confounding factors such as the potential existence of primaquine-resisted Plasmodium, which may exaggerate the identification of relapsed vivax malaria patients. Finally, although the CYP2D6 enzyme activity was analyzed by the method of assigning the activity score of zygotic allelic recently recommended by Caudle et al [33], this study has defined more CYP2D6 gene locus polymorphisms. Since there is no benchmark standard to scores the activity of these loci polymorphisms, the predicted CYP2D6 enzyme activity in this study may not be completely homogenous with the findings of Caudle et al.

\section{Conclusion}

In conclusion, the prediction method of CYP2D6 enzyme activity based on the sequencing of whole gene coding region of CYP2D6 preliminarily revealed the CYP2D6 gene mutation polymorphisms in relapsed $P$. vivax patients who showed poor curative effect of primaquine in Yunnan. This study ushers in the analysis of genetic correlation factors in relapsed $P$. vivax patients in China. The important findings are as follows: (1) Among the multiple mutations of CYP2D6 gene, the incage mutations at three loci, including 
c.408, c.886 and c. 1457, are most closely related to decreased CYP2D6 enzyme activity. The mutations at c.408 and c.1457 induce to the compromised CYP2D6 enzyme activity, yet whether such decrease is aggravated by mutation of c.886 requires a larger sample size for further validation. (2) The key to predict CYP2D6 enzyme activity by genotype is to define the genotype accurately and accordingly, based on which to study the genetic correlation between genotype and enzyme activity phenotype with reasonable sample size. Because the whole gene sequencing method can fully identify the mutation loci, it is a promising molecular epidemiological method for the investigation of CYP2D6 gene mutation polymorphism.

\section{Abbreviations}

WHO: World Health Organization; CYP2D6: Cytochrome P450, family 2, subfamily D, polypeptide 6; G6PD: Glucose-6-Phosphate Dehydrogenase; CDS: coding DNA sequence; PCR: polymerase chain reaction; PM: Poor metabolizer; IM: Intermediate metabolizer; NM: Normal metabolizer; UM: Ultra-rapid metabolizer; pvcsp: Plasmodium vivax circumsporozoite protein; Hap: haplotype; $\pi$ : Diversity index; He: Expected heterozygosity; OR: odds ratio;

\section{Declarations}

\section{Acknowledgements}

We appreciate the support from the Centers for Disease Control and Prevention in states/cities and counties such as Dehong, Baoshan, Kunming, Pu'er, Lincang, Dali, Nujiang, Lijiang, Xishuangbanna, Yuxi, Chuxiong, Honghe, Zhaotong, Diqing, Qujing, and Whenshan.

\section{Authors' contributions}

$\mathrm{HH}$ was responsible for the gene testing, study design, statistics and analysis of the data, and wrote the manuscript; YDong performed coordination of all project, study design, analysis of the data and the manuscript guidance and revision. SL participated in the study design; YX, YL, YDeng, MC, CZ performed the collection blood samples and microscopy examination. All authors read and approved the final draft of the manuscript.

\section{Funding}

Supported by National Science Foundation (No. 81660559,81960579囚, the Youth Project of Yunnan Province Basic Research Program (No. 2017FD007), and Research institutions in medical and health units of Yunnan Province Research projects $\llbracket N o .2018$ NS0180区

\section{Availability of data and materials}

Not applicable. 


\section{Ethics approval and consent to participate}

The study was approved by Yunnan Institute of Parasitic Diseases and by the Ethical Committee. Genetic testing experiment, etc. were performed on stored blood samples obtained as part of routine diagnostic work-up patients with fever suspected of malaria. Although the absence of risk and the anonymous data processing are ensured among the study, consent from potential malaria patients need to be obtained during collecting blood samples. Demographic, clinical and epidemiological information of each fever patient will be collected. Database access will be restricted by password, and Yunnan Institute Parasitic Diseases will not allow retrieving and saving the personal identification information into the project database. It is committed not to provide information about the patient to any person unrelated to the study.

\section{Consent for publication}

All authors provided their consent for the publication of this report.

\section{Competing interests}

The authors declare that they have no competing interests.

\section{Author details}

${ }^{1}$ School of Basic Medical Sciences, Dali University, Dali 667000, China. ${ }^{2}$ Yunnan Institute of Parasitic Diseases Control, Yunnan Provincial Key Laboratory of Vector-borne Diseases Control and Research, Yunnan Centre of Malaria Research, Academician Workstation of Professor JinNingyi, Pu'er Academician Workstation of Professor Lubin Jiang, Pu'er, 665000, China

\section{References}

1. WHO. World malaria report 2018. Geneva: World Health Organization; 2018. https://www.who.int/malaria/publications/world-malaria-report-2018/en/. Accessed 10 Mar 2020.

2. Recker M, Bull PC. Recent advances in the molecular epidemiology of clinical malaria. F1000Res. 2018; 7.

3. Thuy-Nhien N, Tuyen NK, Tong NT, Vy NT, Thanh NV, Van HT, et al. K13 Propeller Mutations in Plasmodium falciparum Populations in Regions of Malaria Endemicity in Vietnam from 2009 to 2016.Antimicrob Agents Chemother. 2017;61.

4. Dong Y, Wang J, Sun A, Deng Y, Chen M, Xu Y, et al. Genetic association between the Pfk13 gene mutation and artemisinin resistance phenotype in Plasmodium falciparum isolates from Yunnan Province, China. Malar J. 2018; 17:478.

5. WHO Malaria Policy Advisory Committee and Secretarial. Malaria Policy Advisory Committee to the WHO: conclusions and recommendations of September 2012 meeting. Malar J. 2012; 11:424. 
6. Feng J, Zhang L, Huang F, Yin ZH, Tu H, Xia ZG, et al. Ready for malaria elimination: zero indigenous case reported in the People's Republic of China. Malar J. 2018; 17:315.

7. Cao J, Liu YB, Cao YY, Zhu GD, Zhou SS. Sustained challenge to malaria elimination in China: imported malaria. Chin J Parasitol Parasit Dis. 2018,36(02):93-96.

8. Zhang L, Feng J, Tu H, Xia ZG, Zhou SS. Challenges in malaria elimination the epidemiological characteristics of Plasmodium vivax in China from 2011 to 2018. Chin J Parasitol Parasit Dis. 2019; 37:532-538.

9. Bennett JW, Pybus BS, Yadava A, Tosh D, Sousa JC, McCarthy WF, et al. Primaquine failure and cytochrome P-450 2D6 in Plasmodium vivax malaria. N Engl J Med. 2013; 369: 1381-2.

10. Marcsisin SR, Reichard G, Pybus BS. Primaquine pharmacology in the context ofCYP2D6 pharmacogenomics: Current state of the art. Pharmacol Ther. 2016; 161:1-10.

11. Vale N, Moreira R, Gomes P. Primaquine revisited six decades after its discovery. Eur J Med Chem. 2009; 44:937-53.

12. Pybus BS, Sousa JC, Jin X, Ferguson JA, Christian RE, Barnhart R, et al. CYP450 phenotyping and accurate mass identification of metabolites of the 8-aminoquinoline, anti-malarial drug primaquine. Malar J. 2012; 11:259.

13. Wen ZG, An L, Lin F, Wang QZ. Advance in clinical application of CYP2D6 genetic polymorphisms. J Mol Diagn Ther. 2013; 67-72.

14. Zeng R, Mao XB, Liu X, Huang J. Analysis of CYP2D6 gene polymorphism of Chinese population in Hubei. Int J Lab Med. 2018; 39:1540-1542+1546.

15. Twist GP, Gaedigk A, Miller NA, Farrow EG, Willig LK, Dinwiddie DL, et al. Constellation: a tool for rapid, automated phenotype assignment of a highly polymorphic pharmacogene, CYP2D6, from whole-genome sequences. NPJ Genom Med. 2016; 1:15007.

16. Baird JK, Battle KE, Howes RE. Primaquine ineligibility in anti-relapse therapy of Plasmodium vivax malaria: the problem of G6PD deficiency and cytochrome P-450 2D6 polymorphisms. Malar J. 2018; $17: 42$.

17. Nelwan EJ, Ekawati LL, Tjahjono B, Setiabudy R, Sutanto I, Chand K, et al. Randomized trial of primaquine hypnozoitocidal efficacy when administered with artemisinin-combined blood schizontocides for radical cure of Plasmodium vivax in Indonesia. BMC Med. 2015; 13:294.

18. Sutanto I, Tjahjono B, Basri H, Taylor WR, Putri FA, Meilia RA, et al. Randomized, open-label trial of primaquine against vivax malaria relapse in Indonesia. Antimicrob Agents Chemother. 2013; 57:1128-35.

19. Baird JK, Louisa M, Noviyanti R, Ekawati L, Elyazar I, Subekti, et al. Association of Impaired Cytochrome P450 2D6 Activity Genotype and Phenotype With Therapeutic Efficacy of Primaquine Treatment for Latent Plasmodium vivax Malaria. JAMA Netw Open. 2018; 1: e181449.

20. Griese EU, Zanger UM, Brudermanns U, Gaedigk A, Mikus G, Morike K, et al. Assessment of the predictive power of genotypes for the in-vivo catalytic function of CYP2D6 in a German population. Pharmacogenetics. 1998; 8:15-26. 
21. McElroy S, Sachse C, Brockmoller J, Richmond J, Lira M, Friedman D, et al. CYP2D6 genotyping as an alternative to phenotyping for determination of metabolic status in a clinical trial setting. AAPS PharmSci. 2000; 2: E33.

22. Gaedigk A, Bradford LD, Marcucci KA, Leeder JS. Unique CYP2D6 activity distribution and genotypephenotype discordance in black Americans. Clin Pharmacol Ther. 2002; 72:76-89.

23. Lindpaintner K. Pharmacogenetics and the future of medical practice. Br J Clin Pharmacol. 2002; 54:221-30.

24. Crews KR, Gaedigk A, Dunnenberger HM, Klein TE, Shen DD, Callaghan JT, et al. Clinical Pharmacogenetics Implementation Consortium (CPIC) guidelines for codeine therapy in the context of cytochrome P450 2D6 (CYP2D6) genotype. Clin Pharmacol Ther. 2012; 91:321-326.

25. Crews KR, Gaedigk A, Dunnenberger HM, Leeder JS, Klein TE, Caudle KE, et al. Clinical Pharmacogenetics Implementation Consortium guidelines for cytochrome P450 2D 6 genotype and codeine therapy: 2014 update. Clin Pharmacol Ther. 2014; 95:376-382.

26. De Gregori M, Allegri M, De Gregori S, Garbin G, Tinelli C, Regazzi M, et al. How and why to screen for CYP2D6 interindividual variability in patients under pharmacological treatments. Curr Drug Metab. 2010; 11:276-82.

27. Sistonen J, Fuselli S, Levo A, Sajantila A. CYP2D6 genotyping by a multiplex primer extension reaction. Clin Chem. 2005; 51:1291-1295.

28. Sistonen J, Sajantila A, Lao O, Corander J, Barbujani G, Fuselli S. CYP2D6 worldwide genetic variation shows high frequency of altered activity variants and no continental structure. Pharmacogenet Genomics. 2007; 17:93-101.

29. Zanger UM, Raimundo S, Eichelbaum M. Cytochrome P450 2D6: overview and update on pharmacology, genetics, biochemistry. Naunyn Schmiedebergs Arch Pharmacol. 2004; 369:23-37.

30. Gaedigk A, Simon SD, Pearce RE, Bradford LD, Kennedy MJ, Leeder JS. The CYP2D6 activity score: translating genotype information into a qualitative measure of phenotype. Clin Pharmacol Ther. 2008; 83:234-42.

31. Kamenski G, Ayazseven S, Berndt A, Fink W, Kamenski L, Zehetmayer S, et al. Clinical Relevance of CYP2D6 Polymorphisms in Patients of an Austrian Medical Practice: A Family Practice-Based Observational Study. Drugs Real World Outcomes. 2019. doi:10.1007/s40801-019-00177-4

32. Gaedigk A, Sangkuhl K, Whirl-Carrillo M, Klein T, Leeder JS. Prediction of CYP2D6 phenotype from genotype across world populations. Genet Med. 2016. doi: 10.1038/gim.2016.80

33. Caudle KE, Sangkuhl K, Whirl-Carrillo M, Swen JJ, Haidar CE, Klein TE, et al. Standardizing CYP2D6 genotype to phenotype translation: consensus recommendations from the clinical pharmacogenetics implementation consortium and dutch pharmacogenetics Working Group. Clin Transl Sci.2019. doi: $10.1111 /$ cts. 12692

34. Zhang RY, Yang Y, Zou LY, Pei KL, Jing SC, Wang RF, et al『 The Study on CYP2D6, CYP3A5, CYP1A2 Genetic Polymorphism in Chinese Han Population》 Journal of Psychiatry.2018;31:129-132. 
35. Swadi AA, Mohammad BI, Hadi NR. Correlation of CYP2D6 allelic polymorphism to outcome of acute coronary syndrome in mid-Euphrates Iraqi patients on metoprolol therapy. Gene. 2019; 703:112-119.

36. Dong $Y$, Deng $Y, X u Y C$, Chen MN, Mao XH, Sun AM, et al. Analysis of genes associated with antifolate drug resistance in Plasmodium vivax from different infection sources. Chin J Parasitol Parasit Dis. 2018; 36:203-11.

37. Gaedigk A, Ingelman-Sundberg M, Miller NA, Leeder JS, Whirl-Carrillo M, Klein TE, et al. The Pharmacogene Variation (PharmVar) Consortium: Incorporation of the Human Cytochrome P450 (CYP) Allele Nomenclature Database. Clin Pharmaco ITher. 2018; 103:399-401.

38. Dong AN, Ahemad N, Pan Y, Palanisamy UD, Yiap BC, Ong CE. Functional and structural characterisation of common cytochrome P450 2D6 allelic variants-roles of Pro34 and Thr107 in catalysis and Inhibition. Naunyn Schmiedebergs Arch Pharmacol. 2019; 392:1015-1029.

39. Dong Y, Liu SP, Xu YC, Liu Y, Deng Y, Chen MN. Mutations and predicted structure change of G6PD isolated from a patient with primaquine-induced hemolysis in Yunnan Province. Chin J Parasitol Parasit Dis. 2019;3 7:399-405.

40. St Jean PL, Xue Z, Carter N, Koh GC, Duparc S, Taylor M, et al. Tafenoquine treatment of Plasmodium vivax malaria: suggestive evidence that CYP2D 6 reduced metabolism is not associated with relapse in the Phase 2b DETECTIVE trial. Malar J. 2016; 15:97.

41. Brasil LW, Rodrigues-Soares F, Santoro AB, Almeida ACG, Kuhn A, Ramasawmy R, et al. CYP2D6 activity and the risk of recurrence of Plasmodium vivax malaria in the Brazilian Amazon: a prospective cohort study. Malar J. 2018; 17:57.

42. Silvino AC, Costa GL, Araujo FC, Ascher DB, Pires DE, Fontes CJ, et al. Variation in human cytochrome P-450 drug-metabolism genes: a gateway to the understanding of Plasmodium vivax relapses. PLoS ONE. 2016; 11: e0160172.

43. Raimundo S, Toscano C, Klein K, Fischer J, Griese EU, Eichelbaum M, et al. A novel intronic mutation, 2988GA, with high predictivity for impaired function of cytochrome P450 2D6 in white subjects. Clin Pharmacol Ther. 2004; 76:128-38.

44. Wang D, Poi MJ, Sun X, Gaedigk A, Leeder JS, Sadee W. Common CYP2D6 polymorphisms affecting alternative splicing and transcription: long-range haplotypes with two regulatory variants modulate CYP2D6 activity. Hum Mol Genet. 2014; 23:268-278.

\section{Publisher's Note}

Springer Nature remains neutral with regard to jurisdictional claims in published maps and institutional affiliations.

\section{Figures}




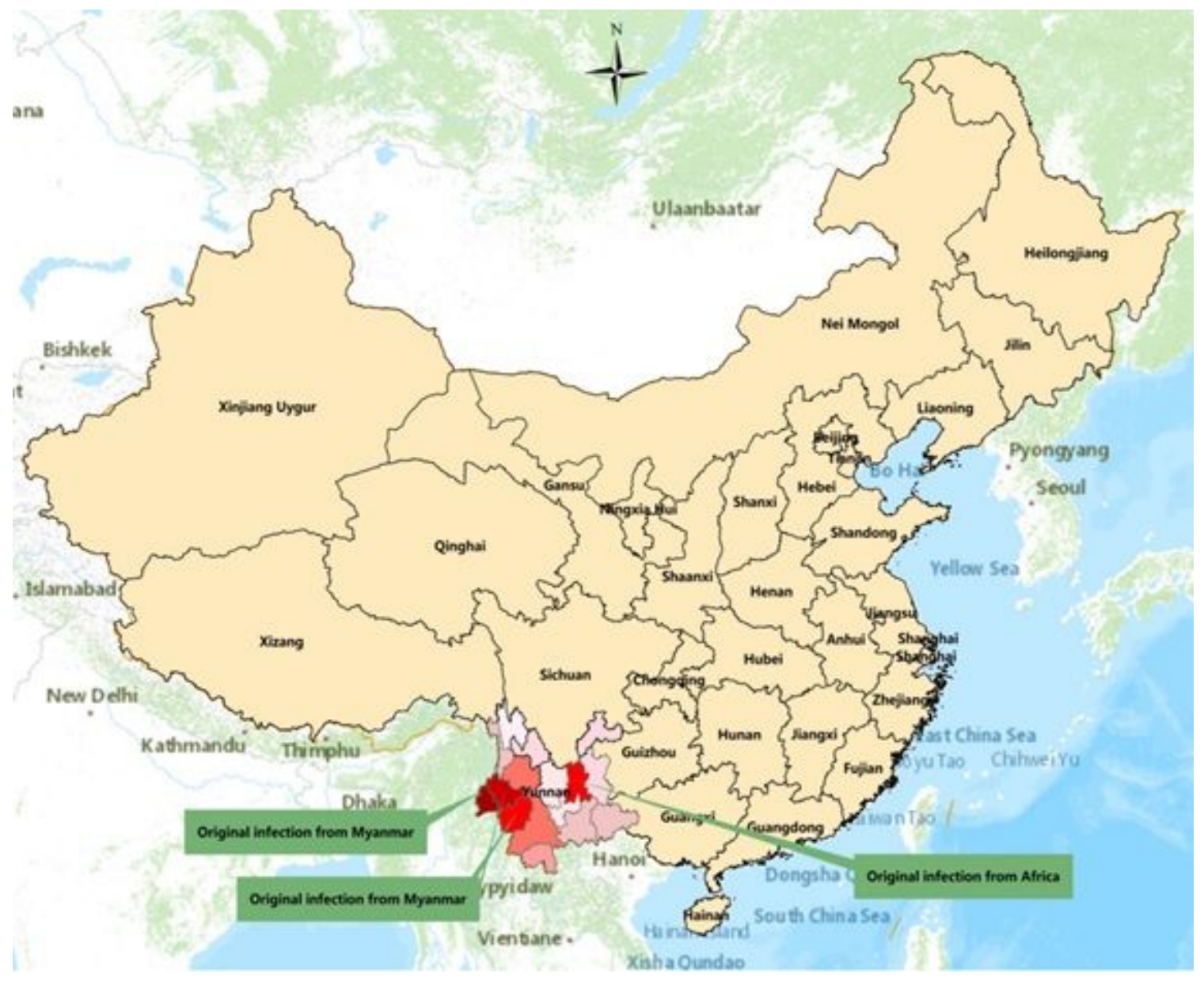

\section{Figure 1}

1 The map of China highlighting the geographic location of Yunnan Province. Different colors represent different administrative areas, and green labels indicate the main source of infection in areas with many imported cases. Note: The designations employed and the presentation of the material on this map do not imply the expression of any opinion whatsoever on the part of Research Square concerning the legal status of any country, territory, city or area or of its authorities, or concerning the delimitation of its frontiers or boundaries. This map has been provided by the authors.
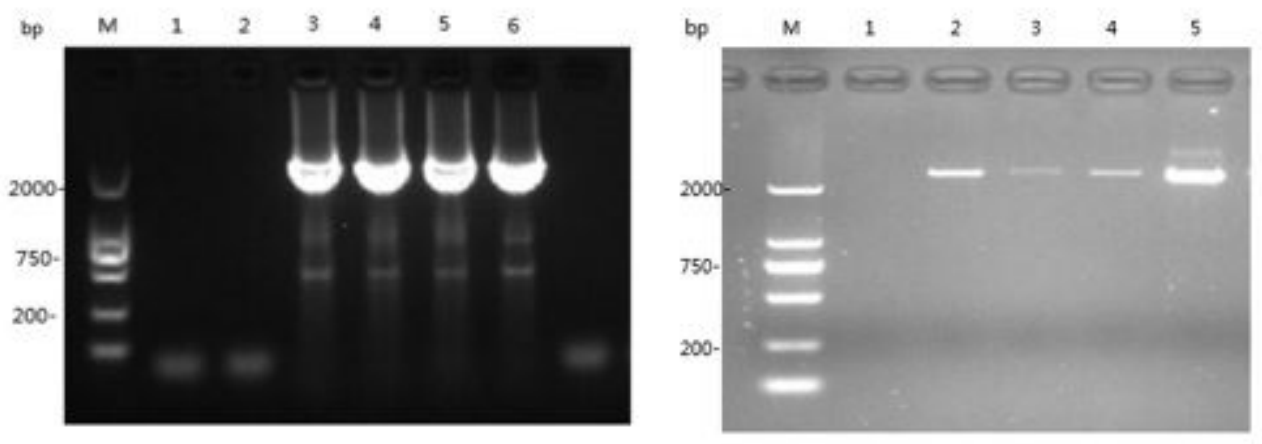
PCR products of CYP2D6 gene fragment. a M: DNA marker; 1: blank control of PCR for the first round; 2: blank control of PCR for the second round; 3-6: The amplification products of exon 1-4 in CYP2D 6 gene; $b$ M: DNA marker; 1: blank control of PCR; 2-5: The amplification products of exon 5-9 in CYP2D6 gene;

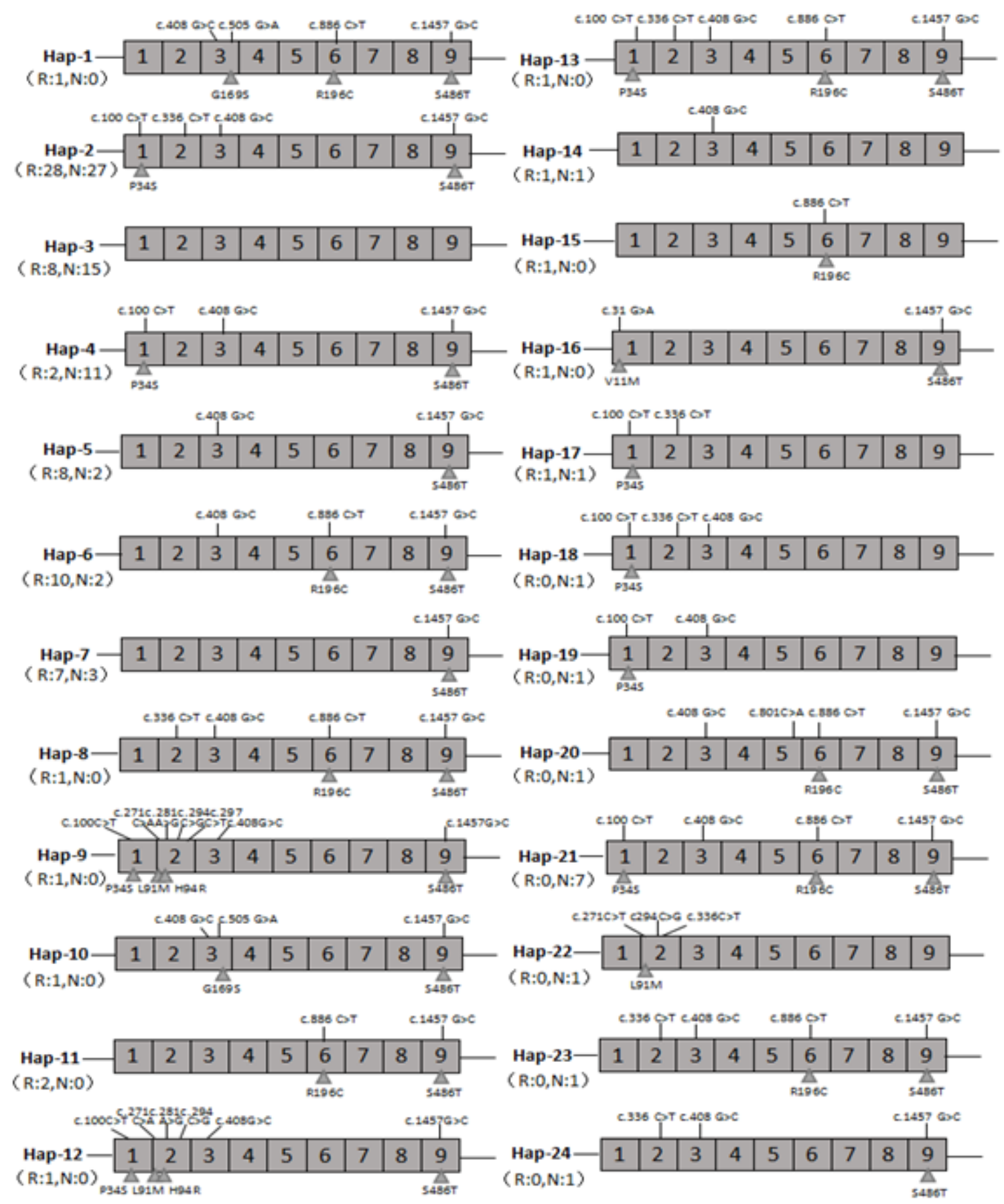

\section{Figure 3}

The 9 exons are indicated by numbered boxes with DNA polymorphisms indicated on top. Predicted amino acid changes are indicated below; no change is synonymous mutation. R: Relapsed cases; N: Nonrelapsed cases.

\section{Supplementary Files}


This is a list of supplementary files associated with this preprint. Click to download.

- Figure1.jpg 